\title{
Designing tailored interventions to address barriers to vaccination
}

\author{
Katrine Habersaat ${ }^{1}$, Noni E MacDonald ${ }^{2}$, Ève Dubé ${ }^{3 \star}$
}

\begin{abstract}
Despite efforts to promote vaccination and make vaccination services easily accessible, vaccination coverage rates remain below the target rate for many vaccines in various jurisdictions. The Tailoring Immunization Programmes (TIP) approach was developed by the World Health Organization Regional Office for Europe to support efforts of countries to achieve high and equitable vaccination uptake. In this Canadian Vaccination Evidence Resource and Exchange Centre (CANVax) series, we present key insights from the TIP planning framework to assist vaccine program planners, policy makers and vaccine providers to identify the interventions that will lead to increased vaccine uptake. The TIP is a phased approach that involves the following: 1) a clear diagnosis of the root cause of low vaccination; 2 ) an intervention based on this understanding; and 3 ) an evaluation of the implementation process and the impact of the interventions. At the provider-patient level, the approaches and insights of the TIP planning framework could inform vaccination consultation by emphasizing the importance of engaging with and listening to the patients and caregivers, and responding to their needs.
\end{abstract}

This work is licensed under a Creative Commons Attribution 4.0 International License.

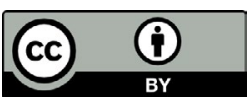

\begin{abstract}
Affiliations
${ }^{1}$ World Health Organization Euro, Geneva, Switzerland

2 Dalhousie University, Halifax, NS ${ }^{3}$ Institut national de la santé publique du Québec, Québec, $\mathrm{QC}$
\end{abstract}

\section{*Correspondence:}

eve.dube@inspq.qc.ca

Suggested citation: Habersaat K, MacDonald NE, Dubé Ė. Designing tailored interventions to address barriers to vaccination. Can Commun Dis Rep 2021;47(3):166-9. https://doi.org/10.14745/ccdr.v47i03a07

Keywords: vaccine acceptance, vaccine hesitancy, interventions, evaluation, Tailoring Immunization Programmes (TIP)

\section{Introduction}

Despite efforts to promote vaccination and make vaccination services easily accessible, vaccination coverage rates remain below the target rate for many vaccines in various jurisdictions. How can we develop effective interventions to increase vaccine acceptance and uptake? This Canadian Vaccination Evidence Resource and Exchange Centre (CANVax) series presents some insights based on the Tailoring Immunization Programmes (TIP) approach (1). The TIP approach was developed by the World Health Organization (WHO) Regional Office for Europe to support countries in their efforts to achieve high and equitable vaccination uptake. The underlying principle of this approach is that it is necessary to understand the barriers to vaccination among the population groups with suboptimal coverage before embarking on any plans for interventions. The key principles guiding TIP approach are highlighted in Figure 1. The TIP is a comprehensive and phased approach that requires the investment of time and resources. Even if your organization does not conduct a full TIP evaluation, the key insights provided in this article will help you to design an effective intervention to enhance vaccine acceptance and uptake.
Figure 1: Values and principles guiding Tailoring Immunization Programmes

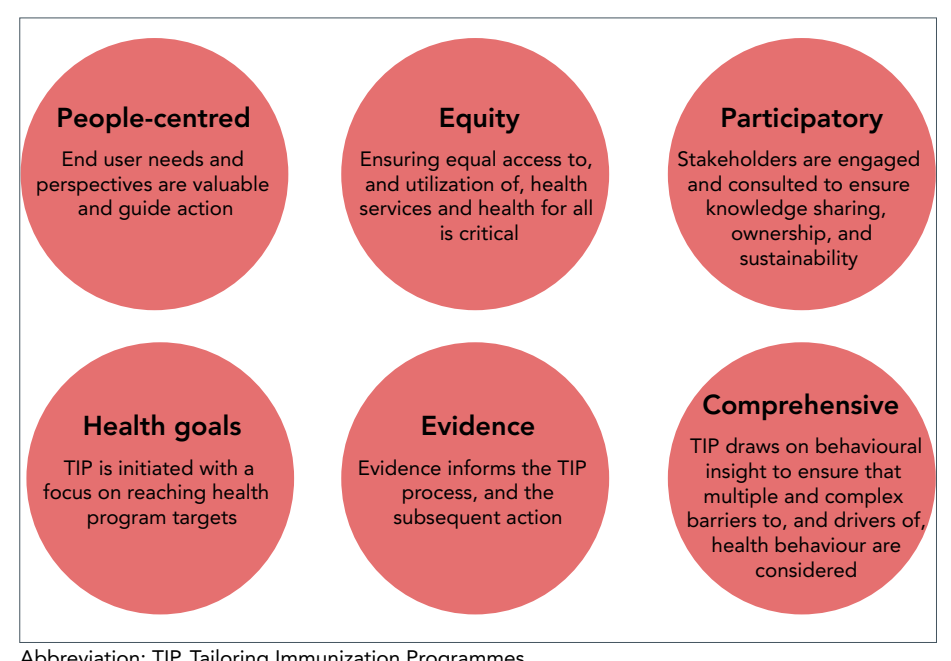

The TIP approach, while designed for use at the national level, is also applicable at the patient-provider level. The TIP approach and resultant insights can inform the planning of vaccine consultations in a healthcare providers' office. 
The objective of this CANVax is to illustrate, through the use of a fictitious case study, how key approaches used by the TIP planning framework could assist vaccine program planners, policy makers and vaccine providers coming up with the right intervention leading to increasing vaccine uptake.

This is the eleventh in a series of articles, produced by CANVaxan online database that supports immunization program planning and delivery. This series includes both the identification of existing resources and the description of the new resources developed by a multidisciplinary group of professionals (2). The article is one of a series and shows how the various aspects of vaccine hesitancy that have been considered to date can be applied to fostering vaccine acceptance.

\section{Canadian case study}

Case study part 1 :

A school-based program of vaccination against the human papillomavirus (HPV) was implemented in your jurisdiction in 2008. After the first year of the program, the vaccine coverage rate was found to be above $80 \%$. However, in the years following the first year, the HPV vaccine coverage rates were found to be declining. To improve the vaccine coverage rate, an educational campaign targeting the parents of students was implemented last year and training sessions for school nurses were offered. Despite these interventions, the HPV vaccine uptake rates are still declining. What can be done?

The WHO TIP approach offers a method to diagnose the barriers to, and drivers of, vaccination in specific subgroups and to design appropriate interventions to address these populations. The TIP approach uses social and behavioural insight methods (i.e. people-centred research and social sciences methods) to design and evaluate interventions for behaviour change. For more information on TIP, see TIP Tailoring Immunization Programmes (2019) (2).

The first step in the process is to understand the problem and explore the reasons behind it to fully understand the barrier(s).

\section{Tailoring Immunization Programmes insight: Diagnose the problem-do not just guess}

Often, the causes of low vaccination coverage rates are not understood, and the interventions are designed based on experts' intuition rather than on actual data.

- "We have tried that in the past and it worked."

- "If only they knew how safe and effective vaccines are, they would vaccinate."
In contrast, the TIP approach emphasizes that the very first step to finding a solution is to have a good understanding of the root cause of the problem. This can do this by

- Looking at the relevant studies conducted in your jurisdiction

- Questioning front-line health providers, the students/ parents/potential recipients, members of the local community and/or other key stakeholders

The aim is to identify the main barriers to, and drivers of, the intended immunization behaviour in the target group:

- Is the problem related to vaccination services? To a lack of awareness? To misinformation in social media? Only by having a good understanding of the causes of the problem will you be able to develop an effective intervention.

Case study part 2:

Interviews were conducted with school nurses and parents to assess their opinions regarding the school-based HPV vaccination program. Findings showed that an important barrier to HPV vaccination in school-based programs was related to the informed consent process. Parents reported that they did not know they needed to sign and return the form to the school nurse to have their child vaccinated. Nurses noted that the short time period between the distribution of informed consent forms to students and the vaccination day prevented them from sending reminders to parents.

\section{Tailoring Immunization Programmes insight: Design the tailored intervention}

Once you have a good understanding of the root cause of the problem, the next step is to design an intervention based on both this understanding and the resources available. If the issues are about access to vaccination services, then interventions aimed to inform people about the risk and benefits of vaccines will not be effective. If lack of awareness is the main cause of under-vaccination, this needs to be addressed first.

Generally, interventions that have multiple components are more effective than single-component interventions. For example, even a simple intervention such as a change in clinic hours requires communication to the community-not just announcing the hours of change on the clinic door. The Behaviour Change Wheel model (see Figure 2) can help inform the design of the intervention to address health behaviours by highlighting the relevant types of interventions, depending on the barriers and drivers identified (2). The TIP approach has adapted this model for vaccination-related concerns (1). Note there are multiple components that need to be considered.

Additional information on effective interventions to increase vaccine acceptance and uptake can be found on the CANVax fact sheets (2). 
Figure 2: Behaviour Change Wheel

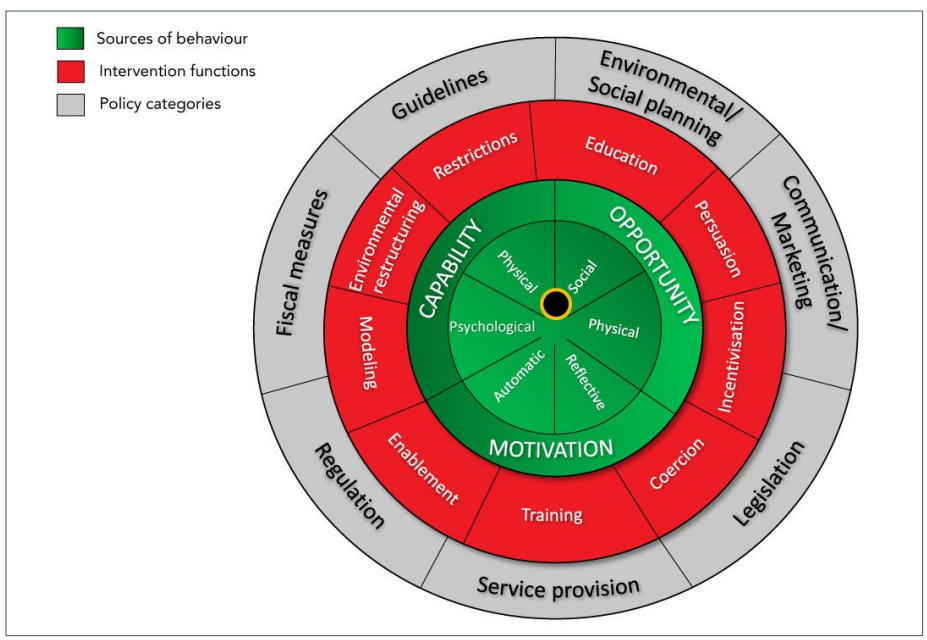

Case study part 3:

The qualitative evaluation has identified "opportunity" barriers related to the organization of vaccination services. An intervention is then designed based on the distribution of informed consent forms to parents by teachers at the beginning of the school year; in addition, an email reminder is sent one month prior to the vaccination day to: 1) remind parents about the upcoming vaccination; 2) ask them to return the signed consent form; and 3) give the contact information of the school nurse in case parents have questions about vaccination. An evaluation of the feasibility and impact of this intervention is ongoing.

\section{Tailoring Immunization Programmes insight: Implementation and Evaluation}

Too often, the work of improving an immunization program stops after the interventions have been implemented. When possible, a good practice is to evaluate the implementation process and the impact of the interventions. Even if you are not conducting a large study, try to evaluate how the interventions were implemented and check whether there was an increase in vaccine uptake. This could be done using regular vaccination program monitoring activities (e.g. coverage assessment before and after the implementation of the intervention). Examples of such evaluation include formal surveys or interviews, or simply by speaking with the people involved in the process to assess the implementation so far and the successes and shortcomings experienced.

\section{Tailoring Immunization Programmes insight: Approaches that healthcare providers could use to increase vaccine uptake among their patients and in their community}

The driving premise of the TIP approach is that to make vaccination a possible, desirable and positive experience, it is important to engage with and listen to the patients and caregivers and to respond to their needs (1). The values and principles of TIP emphasize that end-user needs and perspectives are valued and guide actions (see Figure 1).

- Ask your patient

The underlying principle of the TIP approach is that it is necessary to understand the barriers to vaccination. In the healthcare providers office, this could simply mean exploring why the patient or caregiver is hesitant to get vaccinated. Eliciting the real reasons behind the reluctance would assist the healthcare provider to address the barrier specifically and effectively. An earlier CANVax brief on motivational interviewing provided practical tools and examples how such conversation could play out (3).

- $\quad$ Take the time, work as a team

The TIP approach proposes that the encounter between the patient and the healthcare provider is a critical moment in vaccination decision-making. It is often heard from both providers and patients that vaccination consultations are short and thus provide for only superficial or limited discussions. However, when applying the motivational interviewing techniques (3), it is possible to provide a short and effective counselling about vaccination. With very hesitant patients/ caregivers, more time may be required, so healthcare providers should schedule more time with these patients/caregivers to fully explore barriers and drivers to vaccination.

In many clinics, there are also allied health professionals who are often a great resource as they can take the time to answer patients/caregivers' questions regarding vaccination. It is important that from the time patients enter the clinic-and meet with the office coordinator, then a nurse, then the physician-that the culture and tone is set and consistent. If all the healthcare providers are "singing from the same song sheet", it is more likely that patients/caregivers will be supportive of vaccinations.

- Provide an example to imitate

Healthcare providers demonstrating their vaccination behaviors (e.g. confirm that they vaccinated themselves/their children) and using these behaviours both to promote good vaccination practice among themselves and to set an example for their patients is a TIP recommended activity.

\section{- $\quad$ Share with your peers}

The TIP advocates a formal evaluation process for measuring the impact of newly developed and implemented interventions for increasing vaccine uptake. However, a formal evaluation is not possible or practical in a healthcare providers' office. Instead, taking stock, sharing your experiences with identifying specific barriers and how you addressed them and what strategies 
worked for you, and learning from your colleagues' experiences, can be extremely valuable.

\section{Conclusion}

In conclusion, TIP is a valuable and effective approach to designing interventions to address barriers to vaccination. It is based on the understanding of needs and realities of individuals and communities. Even if you are not doing a formal TIP project, you can apply the key principles guiding TIP (Figure 1) to design your intervention (1).

Key approaches used by the TIP planning framework could assist vaccine program planners, policy makers, as well as vaccine providers in tailoring vaccination services to meet the needs of patients and caregivers, particular groups where increasing vaccine uptake is necessary.

\section{Authors' statement}

$\mathrm{KH}$ - Conceptualization, writing-review and editing NEM - Validation, writing-review and editing

ÈD -Validation, writing-original draft, review and editing

The content and view expressed in this article are those of the authors and do not necessarily reflect those of the Government of Canada.

\section{Competing interests}

None.

\section{Acknowledgements}

Contributions to Canadian Vaccination Evidence Resource and Exchange Centre (CANVax) come from a very wide range of authors, committees, immunization partners, reviewers and especially the CANVax secretariat at Canadian Public Health Association.

\section{Funding}

Dr. MacDonald reports grants from the Public Health Agency of Canada, the Canadian Institutes of Health Research, Nova Scotia Health Authority, IWK Health Authority and the Canadian Immunization Research Network.

Dr. Dubé reports grants from the Public Health Agency of Canada, the Québec Ministry of Health and Social Services, le Fonds de la recherche en Santé du Québec, the Canadian Institutes of Health Research, the Canadian Immunization Research Network and the Social Sciences and Humanities Research Council of Canada.

\section{References}

1. World Health Organization Regional Office for Europe. TIP Tailoring Immunization Programmes (2019). Geneva (Switzerland): WHO; 2019 (accessed 2019-10-29). http://www.euro.who.int/en/publications/abstracts/ tip-tailoring-immunization-programmes-2019

2. Canadian Public Health Association. The Canadian Vaccination Evidence Resource and Exchange Centre. Ottawa (ON): CANVax (accessed 2019-12-15).

https://www.canvax.ca

3. Gagneur A. Motivational interviewing: A powerful tool to address vaccine hesitancy. Can Commun Dis Rep 2020;46(4):93-7. DOI PubMed 